\title{
Identifying the Biosynthetic Gene Cluster for Triacsins with an $\mathbf{N}$-hydroxytriazene Moiety.
}

\author{
Frederick F. Twigg, ${ }^{[a]}$ Wenlong Cai, ${ }^{[a]}$ Wei Huang, ${ }^{[a]}$ Joyce Liu, ${ }^{[b]}$ Michio Sato, ${ }^{\text {[a] }}$ Tynan J. Perez, ${ }^{[c]}$ \\ Jiaxin Geng, ${ }^{[\mathrm{d}]}$ Moriel J. Dror, ${ }^{[a]}$ Ismael Montanez, ${ }^{[\mathrm{c}]}$ Tate L. Tong, ${ }^{[\mathrm{a}]}$ Hyunsu Lee, ${ }^{[\mathrm{c}]}$ and Wenjun \\ Zhang*[a, e]
}

\begin{abstract}
Triacsins are a family of natural products containing an $N$-hydroxytriazene moiety not found in any other known secondary metabolites. Though many studies have examined the biological activity of triacsins in lipid metabolism, the biosynthesis of triacsins has remained unknown. Here, we report the identification of the triacsin biosynthetic gene cluster in Streptomyces aureofaciens ATCC 31442. Bioinformatic analysis of the gene cluster led to the discovery of the tacrolimus producer Streptomyces tsukubaensis NRRL 18488 as a new triacsin producer. In addition to targeted gene disruption to identify necessary genes for triacsin production, stable isotope feeding was performed in vivo to advance the understanding of $N$-hydroxytriazene biosynthesis.
\end{abstract}

Triacsins are a class of acyl-CoA synthetase (ACS) inhibitors consisting of an 11-carbon alkyl chain and a terminal $\mathrm{N}$ hydroxytriazene moiety (Figure 1). They were discovered almost four decades ago in Streptomyces aureofaciens ATCC 31442 through an antibiotic screening program and characterized as vasodilators. ${ }^{[1-2]}$ Being mimics of fatty acids, triacsins were the first ACS inhibitors to be discovered, ${ }^{[3]}$ and their activities in both bacterial and animal models have made them invaluable in the study of lipid metabolism. ${ }^{[4-7]}$ More recently, triacsins have been shown to have an antimalarial activity similar to artemisinin ${ }^{[8]}$ and an antiviral activity against rotaviruses. ${ }^{[9]}$ Though many studies have covered the biologic activity of the triacsins, the biosynthetic origin of this family of compounds remains elusive.

Four triacsin congeners were originally identified which feature a conserved $N$-hydroxytriazene moiety and vary in the unsaturation pattern of their alkyl chains (Figure 1). The $N$-hydroxytriazene moiety, containing three heteroatomheteroatom linkages, is rare in nature and has only been identified in the triacsin family of natural products. Given the prevalence of $\mathrm{N}-\mathrm{N}$ and $\mathrm{N}-\mathrm{O}$ linkages in synthetic drug libraries, there is a great interest in their enzyme catalyzed formation to complement the challenges in synthetic approaches. ${ }^{[10-11]}$ Up to date, multiple classes of enzymes have been characterized to catalyze $\mathrm{N}-\mathrm{O}$ linkages such as cytochrome P450s and $\mathrm{N}$-oxygenases which may be flavindependent or metal-containing. ${ }^{[11]}$ For $\mathrm{N}-\mathrm{N}$ bond formation, few mechanisms have been examined until very recently. One chemical logic goes through the activation of one nitrogen to a more electrophilic species followed by intramolecular addition to a second nucleophilic nitrogen. For example, N5 of ornithine is hydroxylated by a flavindependent enzyme (Ktzl) before the N-N bond formation promoted by a heme enzyme (KtzT) (Figure S1). ${ }^{[12-13]}$ Similarly, N6-hydroxylysine is conjugated to glycine followed by intramolecular nucleophilic attack from the glycine amino group catalyzed by a fusion protein (Spb40) consisting of a cupin and a methionyl-tRNA synthetase-like protein (Figure S1). ${ }^{[14-}$ 15]. An alternative mechanism for N-N bond formation features the reaction of an amine with nitrous acid that is generated through the sequential function of a flavin-dependent enzyme (CreE) on aspartate and a lyase (CreD) (Figure S1). ${ }^{[16-20]}$ For example, the diazo moiety found in cremeomycin is generated through the reaction of an aryl amine with nitrous acid promoted by an acyl-CoA ligase homolog (CreM) (Figure S1). ${ }^{[21]}$ Similarly, the

[a] F. Twigg, W. Cai, W. Huang, M. Sato, M. Dror, T. Tong, Prof. Dr. W. Zhang Department of Chemical and Biomolecular Engineering University of California, Berkeley

2151 Berkeley Way, Berkeley, CA 94704 (USA)

E-mail: wizhang@berkeley.edu

[b] J. Liu

Department of Bioengineering, University of California, Berkeley

2151 Berkeley Way, Berkeley, CA 94704 (USA)

[c] T. Perez, I. Montanez, H. Lee

Department of Chemistry, University of California, Berkeley

2151 Berkeley Way, Berkeley, CA 94704 (USA)

[d] J. Geng

Department of Plant and Microbial Biology

University of California, Berkeley, CA 94704 (USA)

[e] Prof. Dr. W. Zhang

Chan Zuckerberg Biohub

San Francisco, CA 94158 (USA) phosphonohydrazide moiety of fosfazinomycin and the diazo moiety of kinamycin are both formed through the intermediacy of nitrous acid, although the dedicated $\mathrm{N}-\mathrm{N}$ forming enzymes have not been identified in these cases (Figure S1). ${ }^{[22]}$ Compared to these $\mathrm{N}-\mathrm{N}$ containing natural products, triacsins have three consecutive nitrogen atoms in the $\mathrm{N}$-hydroxytriazene moiety, adding complexity to $\mathrm{N}-\mathrm{N}$ bond biogenesis. Investigation of the biosynthesis of triacsins may determine if a known mechanism is used or if yet another chemical logic for $\mathrm{N}-\mathrm{N}$ bond formation exists. 
A) tri Biosynthetic Gene Cluster

S. aureofaciens ATCC 31442

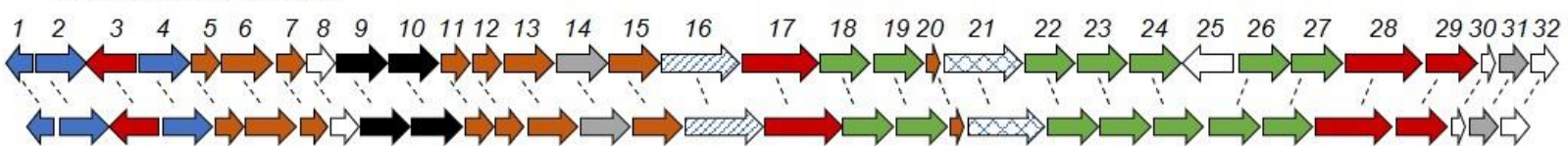

S. tsukubaensis NRRL 18488

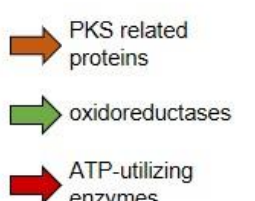

$\Rightarrow$ ATP-utilizin

$\Rightarrow$ decarboxylases

$\square$ transferase/hydrolase
B)

$\Longrightarrow$ hypothetical proteins

$\rightarrow$ transcriptional regulators and transporters

$\Longrightarrow$ CreD homolog

$\Rightarrow$ CreE homolog triacsin A (1)

triacsin B (2)

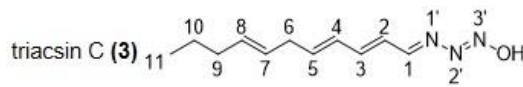

triacsin D (4) c)

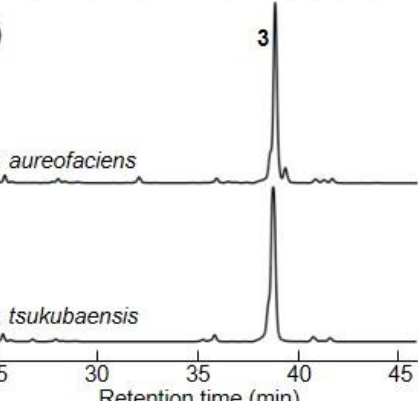

Figure 1. Identification of the triacsin (tri) BGC and a previously unrecognized natural producer. A) Schematic of the tri BGC as discovered in $S$. aureofaciens and the homologous BGC identified in S. tsukubaensis. B) All four of the originally reported triacsin congeners contain an 11-carbon alkyl chain and the unique $N$-hydroxytriazene moiety. C) UV traces (300 nm) confirming the production of 3 in $S$. tsukubaensis. In both strains, the congener $\mathbf{3}$ was produced as the major product and other congeners were not identifiable by UV in these traces.

To identify the potential genetic context for triacsin biosynthesis, the genome of the known triacsin producer, $S$. aureofaciens ATCC 31442, was subjected to Illumina and PacBio sequencing, which resulted in 7.6 M nonredundant bases after assembly of paired sequence reads. The genome was annotated using RAST ${ }^{[23]}$ and biosynthetic gene clusters (BGCs) were predicted using antiSMASH. ${ }^{[24]}$ Genome mining for triacsin BGC candidates was performed by a local BLASTP analysis on the genome using probes such as polyketide synthases (PKSs) which may be involved in the alkyl chain formation, or enzymes from known N-N bond formation pathways such as KtzT, CreE and CreD. While no homologs were found for KtzT, our bioinformatic search turned up two putative BGCs encoding both CreE and CreD homologs (Figures 1 and S2). Cluster 1 spanned $30 \mathrm{~kb}$ and contained 21 open reading frames (ORFs). Cluster 2 spanned $39 \mathrm{~kb}$ and contained 32 ORFs. A targeted gene disruption deleting a $9 \mathrm{~kb}$ multigene region from cluster 1 did not affect triacsin production (Figures S3 and S4). In comparison, a similar multigene disruption in cluster 2 abolished the production of all triacsins (Figure S4). We then assign the cluster 2, designated tri1-32, as the putative triacsin BGC.

To further confirm the importance of this BGC in triacsin biosynthesis, we used an architecture search with the MultiGeneBlast too|[25] looking for regions of homology in or surrounding the tri BGC within published genomes. Two additional microbes were identified containing BGCs highly homologous to the tri BGC (Figures 1 and S5); the marine actinomycete Salinispora arenicola CNS-205 (Accession \#: PRJNA17109) ${ }^{[26]}$ and the well-studied tacrolimus producer Streptomyces tsukubaensis NRRL 18488 (Accession \#: PRJNA389523). ${ }^{[27-29]}$ We then cultured these strains to examine their capability in producing triacsin family compounds or potential derivatives. While $S$. arenicola did not appear to produce triacsins in the tested conditions, triacsins were confirmed to be produced by $S$. tsukubaensis through UV and liquid chromatography-high resolution mass spectrometry (LC-HRMS) analyses (Figures 1 and S6). Similar to $S$. aureofaciens, $S$. tsukubaensis produced all four known triacsin congeners with triacsin $C$ (3) being the major metabolite. Careful analysis of $S$. tsukubaensis cultures identified four additional compounds with UV spectra similar to 3 or triacsin $D(4)$ and the same molecular formulas of $\mathrm{C}_{11} \mathrm{H}_{17} \mathrm{~N}_{3} \mathrm{O}_{2}$ indicated by HRMS, suggesting that they were hydroxylated triacsin derivatives (5-8) (Figure S7). These results revealed for the first time that $S$. tsukubaensis is a triacsin producer, and strongly support that the identified tri BGC is related to triacsin biosynthesis since no other homologous BGCs were identified between $S$. aureofaciens and $S$. tsukubaensis. Further sequence analysis showed that the triacsin BGC architecture in $S$. tsukubaensis is nearly identical to that in $S$. aureofaciens except the lack of tri25, the gene encoding a putative transposase. By comparing the sequences around the two clusters, the boundary of the tri cluster was putatively identified (Figure 1).

Bioinformatic analysis of tri1-32 (Table S1) revealed that in addition to ORFs that encode a CreE, CreD, and transposase homolog, the cluster encodes eight discrete PKS related proteins: a ketoreductase (KR), three ketosynthases (KS), two dehydratases (DH), an acyl carrier protein (ACP) and a phosphopantetheinyl transferase, which could be involved in the alkyl chain biosynthesis. Seven ORFs were identified to encode oxidoreductases, which may be responsible for regioselective unsaturation of alkyl chains observed in various triacsin congeners as well as the $\mathrm{N}$-hydroxytriazene moiety formation. Additional biosynthetic enzymes encoded by the cluster include four ATP-utilizing enzymes, two decarboxylases, two transferase/hydrolase homologs, and three hypothetical proteins. Two putative 


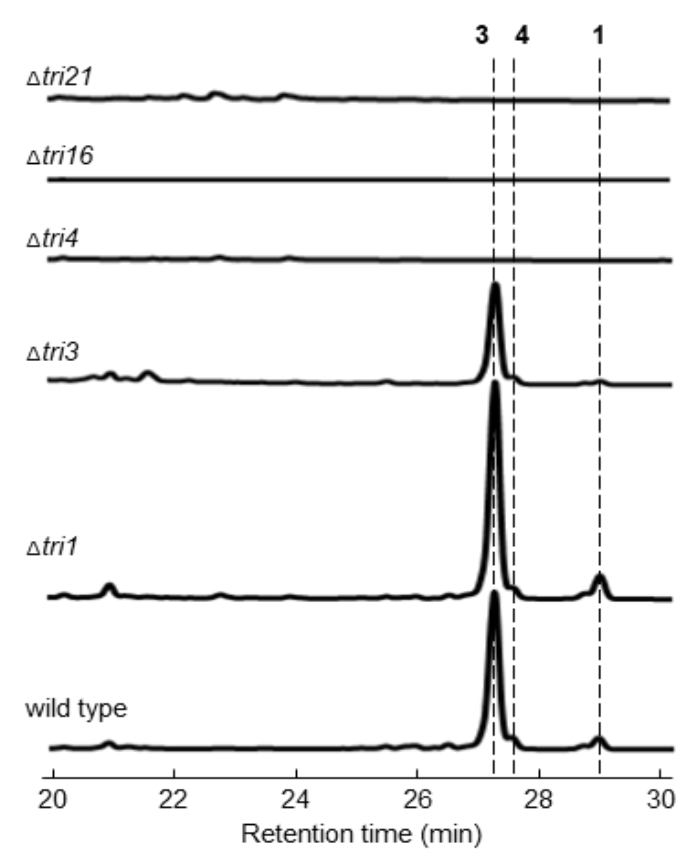

Figure 2. UV traces $(300 \mathrm{~nm})$ demonstrating the effect of selected single gene disruptions on triacsin production in S. aureofaciens. transcriptional regulators and one transporter encoding genes were also identified in the cluster (Figure 1, Table S1). It is notable that tri26-31 are homologous to the gene cassette spb38-43 which was essential for the biosynthesis of the glyoxylate hydrazine unit in s56-p1, ${ }^{[14-15]}$ suggesting that at least one $\mathrm{N}-\mathrm{N}$ bond in triacsins is formed via Tri28, the homolog of Spb40 consisting of a cupin and a methionyltRNA synthetase-like protein. In addition, hydrazinoacetic acid (HAA) could be an intermediate for triacsin biosynthesis which is proposed to be generated through the activities of Tri26-28 from lysine and glycine (Figure S8). ${ }^{[14-15]}$ The third nitrogen in the $N$-hydroxytriazene moiety could be derived from nitrous acid due to the presence of Tri16 and 21, the CreD and CreE homologs that presumably generate nitrous acid from aspartate (Figure S8), however enzymes to promote this $\mathrm{N}-\mathrm{N}$ linkage remain unclear.

In order to probe the necessity of the encoded enzymes in triacsin biosynthesis, we disrupted individual genes for the majority of the ORFs through double crossover in $S$. aureofaciens and/or $S$. tsukubaensis (Figure S3). Triacsin production was completely abolished in $\Delta$ tri5-14, 16-19, 21-22, 26-29, 31-32, demonstrating that these genes were required for triacsin biosynthesis (Figures 2 and S9-10). These results are consistent with the proposed enzymes, including Tri16, 21, 26, 27, 28, which are involved in the $N$ -

hydroxytriazene moiety formation through two distinct mechanisms for N-N bond formation (Figure S8). Surprisingly, the disruption of tri23 and tri24, encoding two putative saccharopine dehydrogenases, had no effect on any triacsin production in S. tsukubaensis, indicating that they are not essential for triacsin biosynthesis (Figure S10). None of the oxidoreductase-encoding gene disruptions led to differential abolishment of triacsin congener production, suggesting that they are not involved in the alkyl chain modification. Additionally, the disruption of tri3 that encodes one of the four ATP-utilizing enzymes in the cluster decreased triacsin titers by about half in $S$. aureofaciens (Figure 2), suggesting that this gene is also non-essential. The possible role of two regulator-encoding genes, tri1 and 4 , was also examined
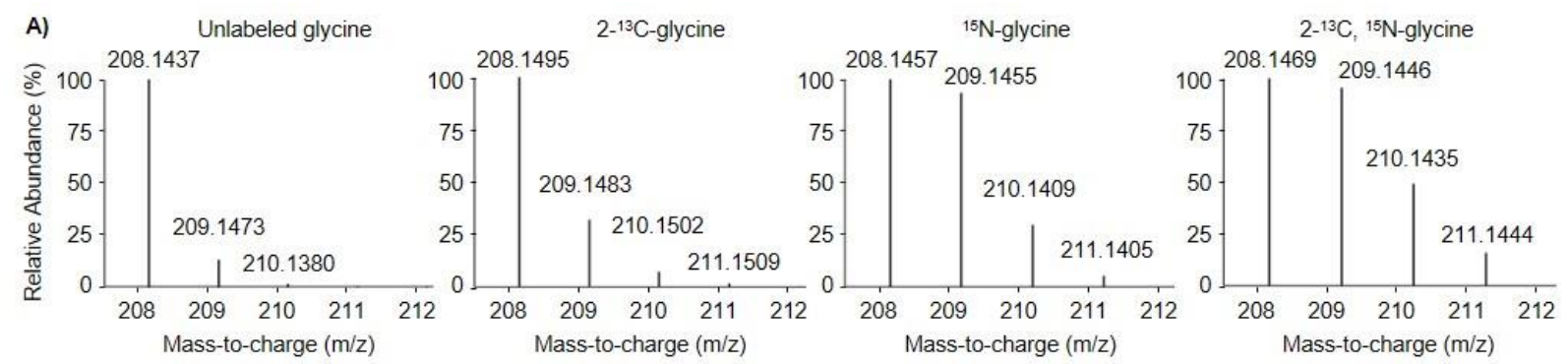

B)
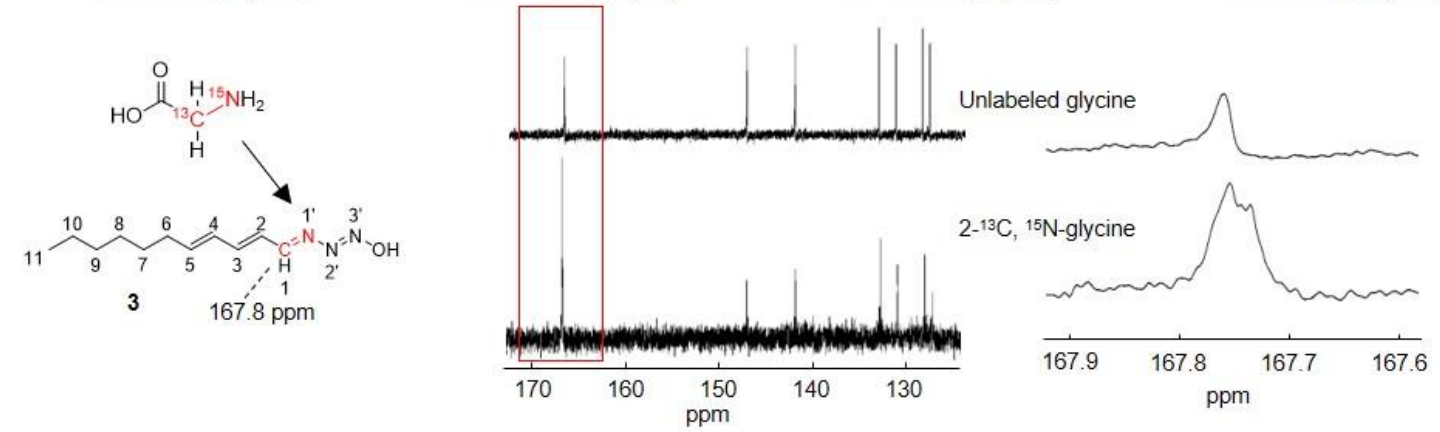

Figure 3. Precursor feeding of isotopically labeled glycine in production of 3 . A) HRMS analysis of $\mathbf{3}$ from cultures provided with varying isotopically labeled glycine substrates. B) ${ }^{13} \mathrm{C}$ NMR spectra of 3 isolated from cultures of $S$. aureofaciens supplied with either $10 \mathrm{mM}$ unlabeled glycine or 2${ }^{13} \mathrm{C},{ }^{15} \mathrm{~N}$-glycine. Close inspection on the enriched $\mathrm{C} 1$ signal shows peak splitting, indicative of an adjacent ${ }^{15} \mathrm{~N}$, which is not observed in $\mathbf{3}$ purified from cultures fed unlabeled glycine. 
through gene disruption experiments in $S$. aureofaciens. $\Delta$ tri1 resulted in a small titer increase while $\Delta$ tri4 abolished triacsin production (Figure 2), suggesting that the encoded proteins are a negative and a positive transcriptional regulator, respectively.

To further support the proposed chemical logic behind the biosynthesis of the $N$-hydroxytriazene moiety (Figure S8), we performed labeled precursor feeding studies to probe the utilization of aspartate, nitrite, and glycine in triacsin biosynthesis. Aspartate was proposed to be the substrate for Tri16 and 21 to form nitrous acid and thus one of the nitrogen atoms in triacsins could be derived from the amino group of aspartate. The feeding of ${ }^{15} \mathrm{~N}$-aspartate resulted in an enrichment of singly labeled 3 (Figure S11). Additionally, the feeding of ${ }^{15} \mathrm{~N}$-nitrite also resulted in an enrichment of singly labeled 3 (Figure S11), consistent with the proposed intermediacy of nitrous acid. Glycine, on the other hand, was proposed to be the substrate for Tri28 to form a hydrazine-containing lysine-glycine adduct, which then undergoes an oxidative cleavage to yield HAA and 2-aminoadipate 6-semialdehyde catalyzed by Tri27, an FAD-dependent Damino acid oxidase homolog (Figure S8). The feeding of ${ }^{15} \mathrm{~N}$-glycine resulted in a significant enrichment of singly labeled 3 (Figure 3A), consistent with the proposed function of Tri28. We next fed $2-{ }^{13} \mathrm{C}$-glycine to further investigate the fate of the $\alpha$-carbon of glycine in triacsin biosynthesis, considering that all triacsins contain an 11-carbon alkyl chain but PKSs typically generate even-number carbon chains from malonyl-CoAs. The feeding of $2-{ }^{13} \mathrm{C}-\mathrm{glycine}$ resulted in a weak enrichment of singly labeled 3, far less than the feeding of ${ }^{15} \mathrm{~N}$-glycine (Figure $3 \mathrm{~A}$ ). In addition, the enrichment of singly labeled 3 obtained from the feeding of $2-{ }^{13} \mathrm{C},{ }^{15} \mathrm{~N}$-glycine and ${ }^{15} \mathrm{~N}$-glycine was comparable (Figure $3 \mathrm{~A}$ ), making the incorporation of the $\alpha$-carbon of glycine in triacsin inconclusive. We then scaled up the triacsin-producing cultures supplied with $2-{ }^{13} \mathrm{C},{ }^{15} \mathrm{~N}$-glycine followed by purification and NMR analysis of 3 . Comparison of the ${ }^{13} \mathrm{C}$ NMR spectra of 3 produced upon the feeding of $2-{ }^{13} \mathrm{C},{ }^{15} \mathrm{~N}$-glycine and unlabeled glycine showed that the $\mathrm{C} 1$ position was enriched (Figure 3B and S12-13), indicating that this carbon could be derived from the a-carbon of glycine. In addition, splitting of the $\mathrm{C} 1$ signal not witnessed in unenriched 3 (Figure $3 \mathrm{~B}$ ) indicates that the N1' was also enriched, consistent with the hypothesis that the $\mathrm{C}-\mathrm{N}$ bond of glycine could be incorporated into triacsins as an intact unit, likely via the HAA intermediate (Figure S8). The apparent disproportional ratio of carbon and nitrogen incorporation upon $2-{ }^{13} \mathrm{C},{ }^{15} \mathrm{~N}-$ glycine feeding suggests that glycine molecules could further donate their nitrogen atoms to other positions on triacsins.

In summary, we have identified the gene cluster for triacsins

that sets the stage for further deciphering of the enzymatic machinery for biosynthesis of this class of ACS inhibitors with a unique $N$-hydroxytriazene moiety. Our bioinformatics, mutagenesis, and labeled precursor feeding experiments allow a first proposal of the chemical logic for $N$-hydroxytriazene biosynthesis via an intramolecular reaction of electrophilic and nucleophilic nitrogen as well as a nitrous acid dependent N-N bond formation (Figure S8). The identification of the triacsin gene cluster further led to the discovery of an additional triacsin natural producer. Future biochemical study will provide more insight in the timing and mechanisms of the biosynthesis of this intriguing class of natural products and expand our understanding of enzymes that catalyze the unique $N$-hydroxytriazene moiety formation.

\section{Experimental Section}

Full experimental details are available in the Supporting Information.

\section{Acknowledgements}

We thank Paul Jensen (the Scripps Institution of Oceanography) for providing Salinispora arenicola CNS-205, Jeffrey Skerker (UC Berkeley) for helping with genome assembly, and Jeffrey Pelton for assistance with NMR spectroscopic analysis. This research was financially supported by the National Institutes of Health (DP2AT009148), Alfred P. Sloan Foundation, and the Chan Zuckerberg Biohub investigator program.

\section{Keywords: acyl-CoA synthetase inhibitor $\bullet$ biocatalysis $\bullet$ biosynthesis $\bullet$ nitrous acid $\bullet N-N$ bond}

\section{References}

[1] K. Yoshida, M. Okamoto, K. Umehara, M. Iwami, M. Kohsaka, H. Aoki, H. Imanaka, J Antibiot 1982, 35, $151-156$.

[2] H. Tanaka, K. Yoshida, Y. Itoh, H. Imanaka, J Antibiot 1982, 35, 157-163.

[3] S. Omura, J Ind Microbiol 1992, 10, 135-156.

[4] E. J. Hartman, S. Omura, M. Laposata, Prostaglandins 1989, 37, 655-671.

[5] S. Omura, H. Tomoda, Q. M. Xu, Y. Takahashi, Y. Iwai, J Antibiot 1986, 39, 1211-1218.

[6] H. Tomoda, K. Igarashi, J. C. Cyong, S. Omura, J Biol Chem 1991, 266, 4214-4219. 
bioRxiv preprint doi: https://doi.org/10.1101/495424; this version posted December 13, 2018. The copyright holder for this preprint (which was not certified by peer review) is the author/funder. All rights reserved. No reuse allowed without permission.

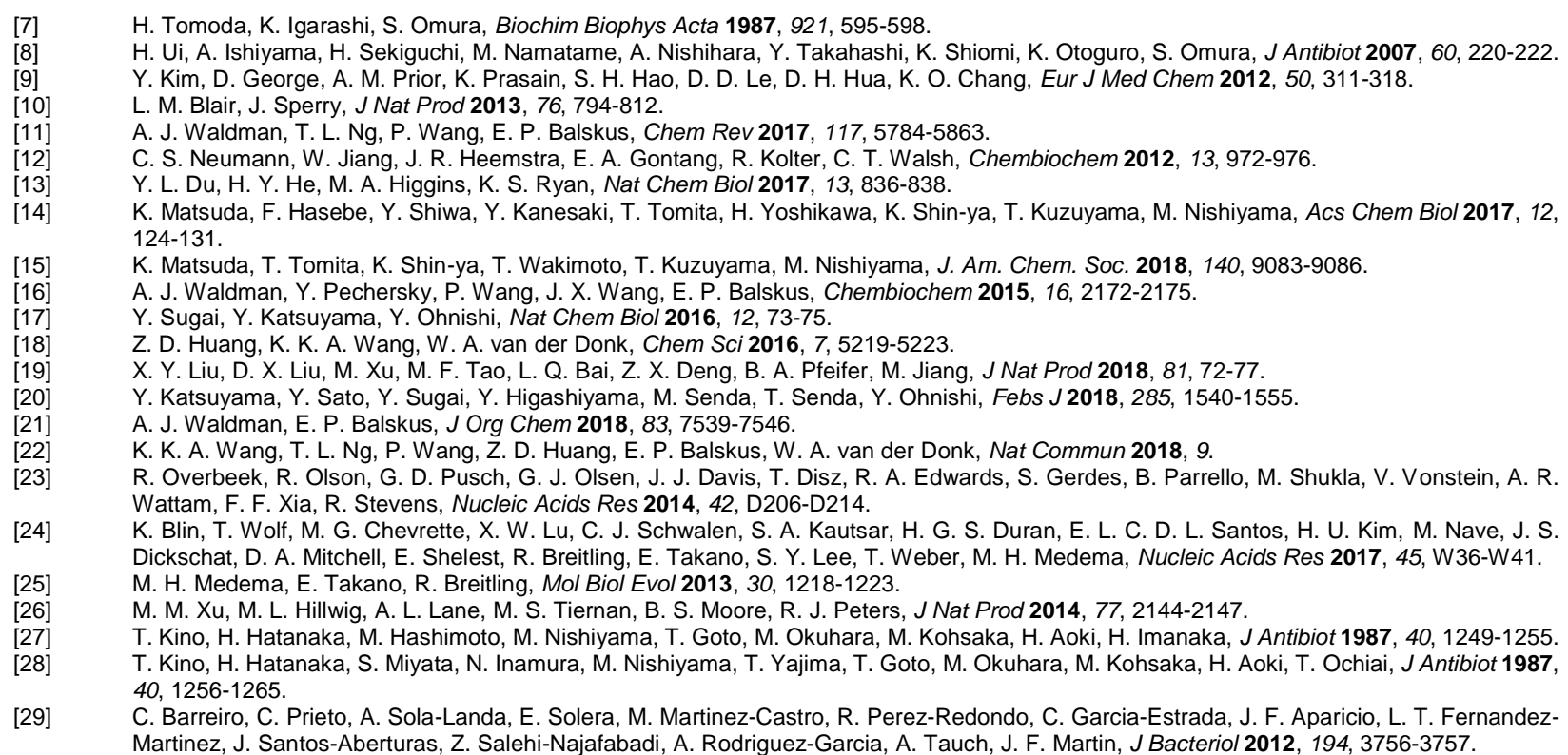

